\title{
Influence of Calcination Parameters over the Morphology and Formation of $\mathrm{Co3}_{4}$ Nanoparticles
}

H. J. Morales-Rodriguez ${ }^{1}$, Alejandro Faudoa-Arzate ${ }^{2}$, W.M. Chávez-Montes ${ }^{3}$, M. Sánchez-Carrillo ${ }^{4}$, Paula Rebeca Realyvazquez-Guevara ${ }^{2}$ and Carlos Arzate-Quintana ${ }^{5}$

${ }^{1}$ Universidad Tecnológica de Chihuahua Sur, Chihuahua, Chihuahua, Mexico, ${ }^{2}$ Tecnológico de Monterrey, Chihuahua, Chihuahua, Mexico, ${ }^{3}$ Universidad La Salle, Chihuahua, Chihuahua, Mexico, ${ }^{4}$ Instituto Tecnológico de Chihuahua, Chihuahua, Chihuahua, Mexico, ${ }^{5}$ Universidad Autónoma de Chihuahua, Chihuahua, Chihuahua, Mexico

\section{Introduction}

Metal oxides are the most important functional materials due to their unique and tunable physical properties have made themselves excellent candidates for electronic and optoelectronic applications. Nanostructured metal oxides have been actively studied due to both scientific interests and potential applications. $\mathrm{Co}_{3} \mathrm{O}_{4}$ is an important antiferromagnetic p-type semiconductor with excellent properties such as gas-sensing, catalytic and electrochemical properties, and has been studied widely for applications in solid-state sensors, electrochromic devices and heterogeneous catalysts as well as lithium batteries $[1,2]$. In this study, we report the influence of calcination parameters over the formation of $\mathrm{Co} 3 \mathrm{O} 4$ nanoparticles.

\section{Methodology}

Cobalt tetraoxide $\left(\mathrm{Co}_{3} \mathrm{O}_{4},\right)$ powders were prepared by the modify Pichini method which consists in a combined process of cobalt complex formation using citric acid (CA) to form stable cobalt complex and in situ polyesterification with poly(ethylene glycol) (PEG) to form the polymeric resin.

In order to obtain cobalt tetraoxide particles with different sizes and morphologies we used calcination at moderate temperatures from 300 to $1100^{\circ} \mathrm{C}$. The calcination of the polymeric resin at a moderate temperature was selected to generate a pure phase multicomponent cobalt oxide. SEM and EDS characterization where performed to study the changes in morphology as well as it composition. MicroRaman Spectroscopy was used to validate our results. The most representative samples are presented in this study.

\section{Results}

Five of the most representative samples are presented in the following section. Figure 1shows the synthesized oxides which were calcinated at $1100^{\circ} \mathrm{C}, 900^{\circ} \mathrm{C}, 600{ }^{\circ} \mathrm{C}$ and $300^{\circ} \mathrm{C}$ respectively. It can be observed that the cobalt oxide consists in a microagglomerated particles, the average diameter ranging from 25 to $5 \mathrm{~mm}$. From EDS we find the sample calcinated at $600{ }^{\circ} \mathrm{C}$ with a $73.4 \mathrm{wt} \%$ Co and $26.6 \mathrm{wt} \%$ $\mathrm{O}$, to validate the EDS results RAMAN study was conducted. In a typical Raman spectrum of cobalt oxide nanoparticles. It can be distinguished five active Raman modes located approximately at $\sim 185.5, \sim 465.3$, $\sim 506.6, \sim 601, \sim 670$, and $\sim 755.5$ [3]. This results are in agreement whit the values of at and $\sim 200.007$, $\sim 482.124, \sim 522.45, \sim 619.86$ and $\sim 688.78 \mathrm{~cm}^{-1}$.

\section{Conclusion}


We can observe the influence of different calcination temperatures, for the highest $\left(1100{ }^{\circ} \mathrm{C}\right)$ the resulting particles are sintered, in the other hand for the lowest temperature $\left(300^{\circ} \mathrm{C}\right)$ the particles were agglomerated, and for both cases wt $\%$ does not agree with $\mathrm{Co}_{3} \mathrm{O}_{4}$. In the medium point at $600^{\circ} \mathrm{C}$ we find a well defined morphology and in agreement with $\mathrm{Co}_{3} \mathrm{O}_{4}$ this can be corroborated by the EDS and RAMAN results.

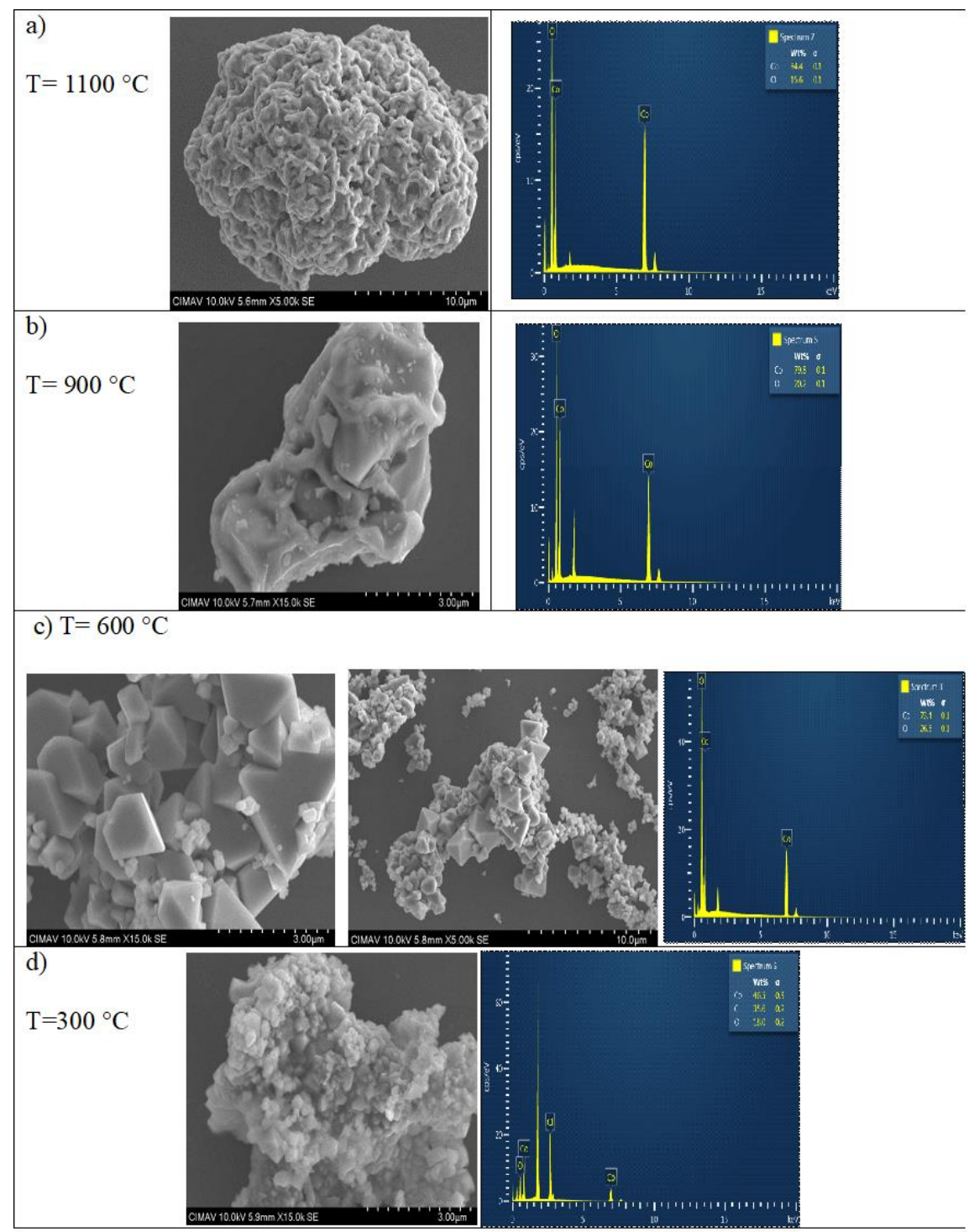

Figure 1. a) , b), c) and d) of $\mathrm{Co} 3 \mathrm{O} 4$ at different calcination temperatures 


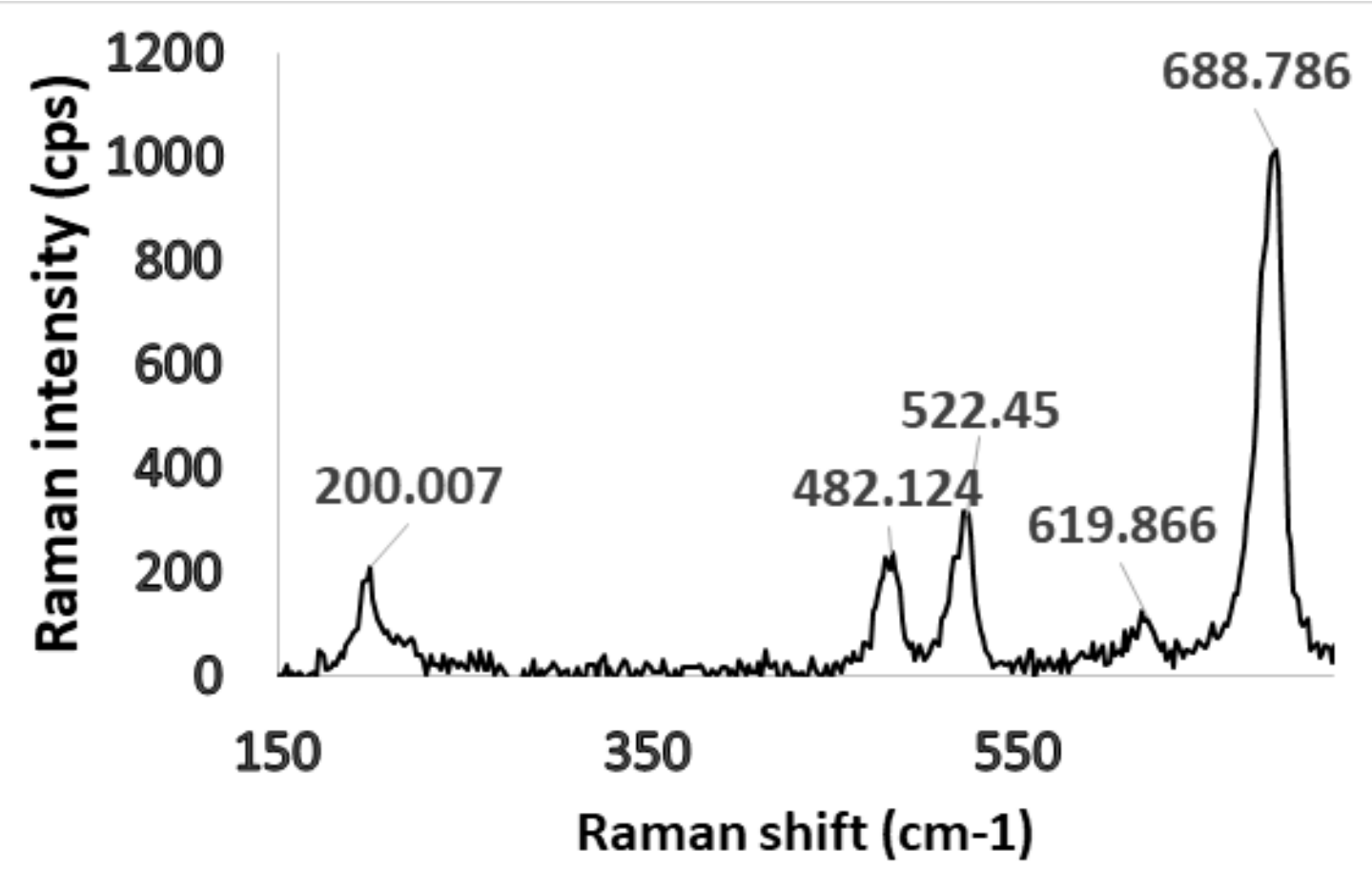

Figure 2. Raman spectrum of $\mathrm{Co} 3 \mathrm{O} 4$ at $600{ }^{\circ} \mathrm{C}$

References

[1] S.Vijayakumar, A. Kiruthika Ponnalagi, S.Nagamuthu, G.Muralidharan (2013). Microwave assisted synthesis of $\mathrm{Co} 3 \mathrm{O} 4$ nanoparticles for high-performance supercapacitors. Electrochimica Acta Volume 106, 1 September 2013, Pages 500-505. doi.org/10.1016/j.electacta.2013.05.121

[2] Edy Saputra, Syaifullah Muhammad, Hongqi Sun, Ha-Ming Ang, Moses Tadé, ShaobinWang, Journal of Colloid and Interface Science Volume 407, 1 October 2013, Pages 467-473. doi.org/10.1016/j.jcis.2013.06.061

[3] A. Diallo, A.C. Beye, T.B. Doyle, E. Park \& M. Maaza (2015) Green synthesis of Co3O4 nanoparticles via Aspalathus linearis: Physical properties, Green Chemistry Letters and Reviews, 8:3-4, 30-36, DOI:10.1080/17518253.2015.1082646 